\title{
Caloric morphisms with respect to radial metrics on $\mathbb{R}^{n} \backslash\{0\}$
}

\author{
KATSUNORI SHIMOMURA*
}

\section{Introduction.}

In our previous paper [5], we defined the notion of caloric morphism, the tranformation which preserve the solutions of heat equation, between Riemannian manifords, and obtained a general characterization theorem. The Appell transformation is the typical example in Euclidean spaces. We also gave some examples of caloric morphism defined on $\mathbb{R} \times\left(\mathbb{R}^{n} \backslash\{0\}\right)(n \geqq 2)$ with respect to radial metrics. In this paper, we shall study such caloric morphisms in more detail. Let $M=\mathbb{R}^{n} \backslash\{0\}$ be the Riemannian manifold with the radial metric of form

$$
g_{i j}=\rho(|x|) \delta_{i j}, \quad i, j=1,2, \ldots, n
$$

where $\rho$ is a positive $C^{\infty}$ function defined on $(0, \infty)$ and $\delta_{i j}$ is Kronecker's delta. The goal of this paper is to determine the caloric morphism defined on a domain in $\mathbb{R} \times M$ of form

$$
f(t, x)=\left(f_{0}(t), \nu(t) R(t) x\right)
$$

or

$$
f(t, x)=\left(f_{0}(t), \frac{\nu(t) R(t) x}{|x|^{2}}\right)
$$

where $\nu(t)$ is a positive $C^{\infty}$-function and $R(t)$ is a $C^{\infty}$-function of orthogonal matrix value. We note that the extistence of the caloric morphism of above forms puts strong restrictions on the metric in the case that the function $\nu$ is not constant. In fact, It is shown that $\rho$ has the form $\rho(r)=p r^{q}$ in such cases. Our main result is formulated in the following theorem.

Partially supported by Grant-in-aid for Scientific Research (C) No. 13640151, Japan Society for the Promotion of Science.

Received May 31, 2003; revised November 18, 2003.

2000 Mathematics Subject Classification. 31B99, 35K99.

Key words . caloric morphism, Riemannian manifold, Appell transformation

* Department of Mathematical Sciences, Ibaraki University, Mito, Ibaraki 310-8512, Japan. e-mail: 
Theorem 1. Let $M=\mathbb{R}^{n} \backslash\{0\}$ be the Riemannian manifold with metric of form $\left(^{*}\right)$ and $(f, \varphi)$ be a caloric morphism of form (a) or (b) defined on a domain $D \subset \mathbb{R} \times\left(\mathbb{R}^{n} \backslash\{0\}\right)$. Then we can determine the forms of $(f, \varphi)$ and $\rho$ as follows. Case 1-a. $n=2$ and $\rho$ is of form $\rho(r)=p r^{-2}(p>0)$ and $f$ is of form (a),

$$
\begin{aligned}
f(t, x) & =\left(t+d, c e^{a t} R_{0}\left(\begin{array}{cc}
\cos b t & -\sin b t \\
\sin b t & \cos b t
\end{array}\right) x\right), \\
\varphi(t, r, \theta) & =C r^{\frac{1}{2} a p} \exp \left(\frac{p}{2} b \theta+\frac{p}{4}\left(a^{2}+b^{2}\right) t\right) .
\end{aligned}
$$

Case 1-b. $n=2$ and $\rho$ is of form $\rho(r)=p r^{-2}(p>0)$ and $f$ is of form (b),

$$
\begin{aligned}
f(t, x) & =\left(t+d, \frac{c e^{a t}}{|x|^{2}} R_{0}\left(\begin{array}{cc}
\cos b t & -\sin b t \\
\sin b t & \cos b t
\end{array}\right) x\right), \\
\varphi(t, r, \theta) & =C r^{-\frac{1}{2} a p} \exp \left(\frac{p}{2} b \theta+\frac{p}{4}\left(a^{2}+b^{2}\right) t\right) .
\end{aligned}
$$

In the cases 1 -a and $1-b, a, b, c, d, C \in \mathbb{R}(c, C>0), R_{0}$ is an orthogonal matrix and $(r, \theta)$ is the polar coordinate of $\mathbb{R}^{2}$.

Case 2-a. $n \geqq 3$ and $\rho$ is of form $\rho(r)=p r^{-2}(p>0)$ and $f$ is of form (a),

$$
f(t, x)=\left(t+d, c e^{a t} R_{0} x\right), \quad \varphi(t, x)=C|x|^{\frac{1}{2} a p} \exp \left(\frac{1}{4} p a^{2} t\right) .
$$

Case 2 -b. $n \geqq 3$ and $\rho$ is of form $\rho(r)=p r^{-2}(p>0)$ and $f$ is of form (b),

$$
f(t, x)=\left(t+d, \frac{c e^{a t}}{|x|^{2}} R_{0} x\right), \quad \varphi(t, x)=C|x|^{-\frac{1}{2} a p} \exp \left(\frac{1}{4} p a^{2} t\right) .
$$

In the cases 2-a and 2-b, $a, c, d, C \in \mathbb{R}(c, C>0)$ and $R_{0}$ is an orthogonal matrix.

Case 3. $\rho$ is of form $\rho(r)=p r^{q}(q \in \mathbb{R}, q \neq-2)$,

$$
\begin{aligned}
& f(t, x)=\left(\frac{c t+d}{a t+b}, \frac{R_{0} x}{|a t+b|^{2 /(q+2)}}\right), \\
& \varphi(t, x)=\frac{C}{|a t+b|^{n / 2}} \exp \left[-\frac{p a|x|^{q+2}}{(q+2)^{2}(a t+b)}\right],
\end{aligned}
$$

where $a, b, c, d, C \in \mathbb{R}(b c-a d=1, C>0)$ and $R_{0}$ is an orthogonal matrix.

Case 4. $\rho(r)$ is any positive $C^{\infty}$-function satisfying $\rho(\nu r)=\lambda \rho(r)$ with some positive constants $\nu$ and $\lambda$,

$$
f(t, x)=\left(\lambda t+d, \nu R_{0} x\right), \quad \varphi(t, x)=C,
$$

where $C>0, d \in \mathbb{R}$ and $R_{0}$ is an orthogonal matrix.

Case 5. $\rho(r)$ is any positive $C^{\infty}$-function satisfying $\rho\left(\frac{\nu}{r}\right)=\frac{\lambda r^{4}}{\nu^{2}} \rho(r)$ with some positive constants $\nu$ and $\lambda$,

$$
f(t, x)=\left(\lambda t+d, \frac{\nu R_{0} x}{|x|^{2}}\right), \quad \varphi(t, x)=C
$$


where $C>0, d \in \mathbb{R}$ and $R_{0}$ is an orthogonal matrix.

Case 6. $\rho$ is any positive $C^{\infty}$-function,

$$
f(t, x)=\left(t+d, R_{0} x\right), \quad \varphi(t, x)=C,
$$

where $C>0, d \in \mathbb{R}$ and $R_{0}$ is an orthogonal matrix.

ACKNOWLEDGMENT. This paper is based on the ideas that the author obtained during his research stay in Katholische Universität Eichstätt. The author wish to express his gratitude for the support and the hospitality he received. The author thanks the referee for his valuable comments.

\section{Notations and Preliminaries.}

Let $(M, g)$ be an $n$-dimensional Riemannian manifold. In this paper, we always consider Riemannian manifolds to be connected. We denote $\Delta_{g}$ the Laplace-Beltrami operator on $(M, g)$ which is given in local coordinates $\left(x_{i}\right)_{i=1}^{n}$ by

$$
\Delta_{g} u=\sum_{i, j=1}^{n} \frac{1}{\sqrt{g}} \frac{\partial}{\partial x_{i}}\left(\sqrt{g} g^{i j} \frac{\partial u}{\partial x_{j}}\right)
$$

where $g=\operatorname{det}\left(g_{i j}\right)$ and $\left(g^{i j}\right)$ denotes the inverse matrix of $\left(g_{i j}\right)$ as usual.

We define the gradient vector field $\nabla_{g} u$ of a $C^{\infty}$-function $u$ by

$$
\nabla_{g} u=\sum_{i, j=1}^{n} g^{i j} \frac{\partial u}{\partial x_{i}} \frac{\partial}{\partial x_{j}}
$$

Then the gradient of the product function $u v$ of two smooth functions $u, v$ is

$$
\nabla_{g}(u v)=u \nabla_{g} v+v \nabla_{g} u
$$

The inner product of two gradients $\nabla_{g} u$ and $\nabla_{g} v$ is given by

$$
g\left(\nabla_{g} u, \nabla_{g} v\right)=\sum_{i, j=1}^{n} g^{i j} \frac{\partial u}{\partial x_{i}} \frac{\partial v}{\partial x_{j}} .
$$

We denote the heat operator $\frac{\partial}{\partial t}-\Delta_{g}$ on $\mathbb{R} \times M$ by $H_{g}$.

A $C^{\infty}$-function $u(t, x)$ defined on a open set $D \subset \mathbb{R} \times M$ is said to be caloric on $D$, if $u$ satisfies the heat equation

$$
H_{g} u=0
$$

on $D$. 
Definition. Let $(M, g)$ be an n-dimensional Riemannian manifold. A pair $(f, \varphi)$ of a $C^{\infty}$-mapping $f$ from a domain $D \subset \mathbb{R} \times M$ to $\mathbb{R} \times M$ and a stricty positive $C^{\infty}$-funcion $\varphi$ defined on $D$ is said to be a caloric morphism, if $f$ and $\varphi$ satisfy the following conditions;

(1) $f(D)$ is a domain in $\mathbb{R} \times M$.

(2) For any subdomain $E \subset f(D)$ and any caloric function $u$ defined on $E$, the function $\varphi(t, x)(u \circ f)(t, x)$ is caloric on $f^{-1}(E)$.

Let $(f, \varphi)$ and $(F, \Phi)$ be caloric morphisms defined on $D$ and $E$, respectively. If $f(D) \subset E$, then it is easily seen that the composition $(F \circ f, \varphi \cdot(\Phi \circ f))$ is a caloric morphism on $D$.

In our previous paper [5], we proved the following characterization theorem for caloric morphism.

Theorem A. Let $(f, \varphi)$ be the pair of a smooth mapping $f$ from $D \subset \mathbb{R} \times M$ to a domain $f(D)$ of $\mathbb{R} \times M$ and a positive smooth function $\varphi$ defined on $D$.

Then the following three conditions are equivalent.

(1) $(f, \varphi)$ is a caloric morphism.

(2) There exists a positive smooth function $\lambda$ on $D$ such that

$$
H_{g}(\varphi \cdot u \circ f)(t, x)=\lambda^{2}(t, x) \varphi(t, x)\left(\left(H_{g} u\right) \circ f\right)(t, x)
$$

holds for any $C^{2}$-function $u$ defined on a subdomain of $f(D)$.

(3) Let $\left(x_{\alpha}\right)_{\alpha=1}^{n}$ be any local coordinate system of $(M, g)$, and let

$$
f=\left(f_{0}, f_{1}, \ldots, f_{n}\right)
$$

be the expression of the mapping $f$ in the coordinate system $\left(t, x_{1}, \ldots, x_{n}\right)$ of the product manifold $\mathbb{R} \times M$. Then $(f, \varphi)$ satisfies the following equations:

$$
H_{g} \varphi=0,
$$

$$
\begin{array}{r}
\frac{\partial f_{\alpha}}{\partial t}=\Delta_{g} f_{\alpha}+2 g\left(\nabla_{g} \log \varphi, \nabla_{g} f_{\alpha}\right)+\sum_{\beta, \gamma=1}^{n} g\left(\nabla_{g} f_{\beta}, \nabla_{g} f_{\gamma}\right)\left(\Gamma_{\beta \gamma}^{\alpha} \circ f\right) \\
\alpha=1, \ldots, n
\end{array}
$$

$$
\nabla_{g} f_{0}=0
$$

$$
g\left(\nabla_{g} f_{\alpha}, \nabla_{g} f_{\beta}\right)=f_{0}^{\prime}(t)\left(g^{\alpha \beta} \circ f\right), \quad \alpha, \beta=1, \ldots, n
$$

where $\Gamma_{\beta \gamma}^{\alpha}=\sum_{l=1}^{n} \frac{1}{2} g^{\alpha l}\left(\frac{\partial g_{\gamma l}}{\partial x_{\beta}}+\frac{\partial g_{\beta l}}{\partial x_{\gamma}}-\frac{\partial g_{\beta \gamma}}{\partial x_{l}}\right)(\alpha, \beta, \gamma=1, \ldots, n)$ is the Christoffel symbol of $(M, g)$.

Remark. In the above theorem, we can replace (E-2) by

$$
\frac{\partial f_{\alpha}}{\partial t}=\Delta_{g} f_{\alpha}+2 g\left(\nabla_{g} \log \varphi, \nabla_{g} f_{\alpha}\right)-f_{0}^{\prime}(t)\left[\left(\Delta_{g} x_{\alpha}\right) \circ f\right], \quad \alpha=1, \ldots, n .
$$




\section{Radial metric on $\mathbb{R}^{n} \backslash\{0\}$.}

In the following, we consider the caloric morphism on the Euclidean space with a radial metric. Let $M=\mathbb{R}^{n} \backslash\{0\}(n \geqq 2)$ and

$$
g_{i j}=\rho(|x|) \delta_{i j}, \quad i, j=1,2, \ldots, n,
$$

where $\rho$ is a positive $C^{\infty}$-function defined on $(0, \infty)$. Then we have

$$
g^{i j}=\frac{1}{\rho(|x|)} \delta_{i j}, \quad g=\rho(|x|)^{n}, \quad \sqrt{g}=\rho(|x|)^{n / 2} .
$$

We can choose the usual cartesian coordinate system as a local coordinate system of $M$. Then the Laplacian and the gradient of a function $u$ are given by

$$
\begin{aligned}
\Delta_{g} u & =\sum_{i=1}^{n} \frac{1}{\rho(|x|)^{n / 2}} \frac{\partial}{\partial x_{i}}\left(\rho(|x|)^{n / 2-1} \frac{\partial u}{\partial x_{i}}\right) \\
& =\frac{1}{\rho(|x|)} \Delta_{x} u+\frac{n-2}{2} \frac{\rho^{\prime}(|x|)}{\rho(|x|)^{2}} \sum_{i=1}^{n} \frac{x_{i}}{|x|} \frac{\partial u}{\partial x_{i}}
\end{aligned}
$$

and

$$
\nabla_{g} u=\frac{1}{\rho(|x|)} \sum_{i=1}^{n} \frac{\partial u}{\partial x_{i}} \frac{\partial}{\partial x_{i}}
$$

respectively. Hence, the inner product of the gradients of two functions $u$ and $v$ is given by

$$
g\left(\nabla_{g} u, \nabla_{g} v\right)=\frac{1}{\rho(|x|)} \sum_{i=1}^{n} \frac{\partial u}{\partial x_{i}} \frac{\partial v}{\partial x_{i}} .
$$

The Laplacian of the coordinate function $x_{\alpha}$ is given by

$$
\Delta_{g} x_{\alpha}=\frac{n-2}{2} \frac{\rho^{\prime}(|x|)}{\rho(|x|)^{2}} \frac{x_{\alpha}}{|x|}, \quad \alpha=1,2, \ldots, n
$$

Let $(f, \varphi)$ be a caloric morphism such that $f$ is of form (a):

$$
f(t, x)=\left(f_{0}(t), \nu(t) R(t) x\right) .
$$

Then $f$ is expressed as

$$
\begin{gathered}
f(t, x)=\left(f_{0}(t), f_{1}(t, x), \ldots, f_{n}(t, x)\right) \\
f_{\alpha}(t, x)=\sum_{j=1}^{n} \nu(t) r_{\alpha j}(t) x_{j}, \quad \alpha=1,2, \ldots, n,
\end{gathered}
$$


where $r_{\alpha j}(t)(\alpha, j=1,2, \ldots, n)$ is the $(\alpha, j)$-element of the orthogonal matrix $R(t)$. Their derivatives are given by

$$
\begin{aligned}
\frac{\partial f_{\alpha}}{\partial t} & =\sum_{j=1}^{n}\left(\nu^{\prime}(t) r_{\alpha j}(t)+\nu(t) r_{\alpha j}^{\prime}(t)\right) x_{j} \\
\frac{\partial f_{\alpha}}{\partial x_{i}} & =\nu(t) r_{\alpha i}(t)
\end{aligned}
$$

for $\alpha, i=1,2, \ldots, n$.

Next, if $(f, \varphi)$ be a caloric morphism such that $f$ is of form (b):

$$
f(t, x)=\left(f_{0}(t), \frac{\nu(t) R(t) x}{|x|^{2}}\right)
$$

then $f_{\alpha}$ is given by

$$
f_{\alpha}(t, x)=\sum_{j=1}^{n} \nu(t) r_{\alpha j}(t) \frac{x_{j}}{|x|^{2}}, \quad \alpha=1,2, \ldots, n
$$

Their derivatives are given by

$$
\begin{aligned}
& \frac{\partial f_{\alpha}}{\partial t}=\sum_{j=1}^{n}\left(\nu^{\prime}(t) r_{\alpha j}(t)+\nu(t) r_{\alpha j}^{\prime}(t)\right) \frac{x_{j}}{|x|^{2}} \\
& \frac{\partial f_{\alpha}}{\partial x_{i}}=\nu(t) r_{\alpha i}(t) \frac{1}{|x|^{2}}-2 \sum_{j=1}^{n} \nu(t) r_{\alpha j}(t) \frac{x_{i} x_{j}}{|x|^{4}}
\end{aligned}
$$

for $\alpha, i=1,2, \ldots, n$.

In the rest of this section, we prepare some lemmas. The first lemma gives the condition for the metric to have a caloric morphism of (a) or (b).

Lemma 1. (1) Let $(f, \varphi)$ be a caloric morphism such that $f$ is of form (a). Then the equation

$$
\rho(\nu(t)|x|)=\frac{f_{0}^{\prime}(t)}{\nu(t)^{2}} \rho(|x|)
$$

holds for all $(t, x) \in D$.

(2) Let $(f, \varphi)$ be a caloric morphism such that $f$ is of form (b). Then the equation

$$
\rho\left(\frac{\nu(t)}{|x|}\right)=\frac{f_{0}^{\prime}(t)|x|^{4}}{\nu(t)^{2}} \rho(|x|)
$$


holds for all $(t, x) \in D$.

Proof. Let $(f, \varphi)$ be a caloric morphism such that $f$ is of form (a). Then by (2) and (4), the equalities

$$
\begin{aligned}
g\left(\nabla_{g} f_{\alpha}, \nabla_{g} f_{\beta}\right) & =\frac{1}{\rho(|x|)} \nu(t)^{2} \sum_{i, j, k=1}^{n} r_{\alpha j}(t) \delta_{j i} r_{\beta k}(t) \delta_{k i} \\
& =\frac{\nu(t)^{2}}{\rho(|x|)} \delta_{\alpha \beta}
\end{aligned}
$$

and

$$
f_{0}^{\prime}(t)\left(g^{\alpha \beta} \circ f\right)(t, x)=f_{0}^{\prime}(t) \delta_{\alpha \beta} \frac{1}{\rho(\nu(t)|x|)}
$$

hold for $\alpha, \beta=1, \ldots, n$, because $R(t)$ is an orthogonal matrix for each $t$. The desired equation

$$
\rho(\nu(t)|x|)=\frac{f_{0}^{\prime}(t)}{\nu(t)^{2}} \rho(|x|), \quad(t, x) \in D
$$

follows from (E-4).

Next let $(f, \varphi)$ be a caloric morphism such that $f$ is of form (b). Then by (2) and (5), the equalities

$$
\begin{aligned}
g\left(\nabla_{g} f_{\alpha}, \nabla_{g} f_{\beta}\right) & =\frac{1}{\rho(|x|)} \frac{\nu(t)^{2}}{|x|^{4}} \sum_{i, j, k=1}^{n} r_{\alpha j}(t)\left(\delta_{j i}-2 \frac{x_{i} x_{j}}{|x|^{2}}\right) r_{\beta k}(t)\left(\delta_{k i}-2 \frac{x_{i} x_{k}}{|x|^{2}}\right) \\
& =\frac{\nu(t)^{2}}{|x|^{4} \rho(|x|)} \delta_{\alpha \beta}
\end{aligned}
$$

and

$$
f_{0}^{\prime}(t)\left(g^{\alpha \beta} \circ f\right)=f_{0}^{\prime}(t) \delta_{\alpha \beta} \frac{1}{\rho(\nu(t) /|x|)}
$$

hold for $\alpha, \beta=1, \ldots, n$, because $R(t)$ is an orthogonal matrix for each $t$. The desired equation

$$
\rho\left(\frac{\nu(t)}{|x|}\right)=\frac{f_{0}^{\prime}(t)|x|^{4}}{\nu(t)^{2}} \rho(|x|), \quad(t, x) \in D
$$

follows from (E-4).

The equations (6) and (7) put strong restrictions on $\rho$ in the case that the function $\nu$ is not constant. In fact, the second lemma shows that $\rho$ has the form $\rho(r)=p r^{q}$ in such cases.

Lemma 2. Let $\rho$ be a positive smooth function defined on an open interval $I \subset(0, \infty)$ and $E$ be a connected open subset of $\mathbb{R} \times\left\{x \in \mathbb{R}^{n} ;|x| \in I\right\}$.

(1) The equation $\rho(\nu(t)|x|)=\frac{\lambda(t)}{\nu(t)^{2}} \rho(|x|)$ holds for all $(t, x) \in E$, where $\lambda$ is a positive smooth function and $\nu$ is a non-constant positive smooth function, if and 
only if there exists a constant $q$ and a positive constant $p$ satisfying $\rho(|x|)=p|x|^{q}$ and $\lambda(t)=\nu(t)^{q+2}$ for all $(t,|x|) \in E$.

(2) The equation $\rho\left(\frac{\nu(t)}{|x|}\right)=\frac{\lambda(t)|x|^{4}}{\nu(t)^{2}} \rho(|x|)$ holds for all $(t, x) \in E, \lambda$ is a positive smooth function and $\nu$ is a non-constant positive function, if and only if $\lambda(t)=1$ and there exists a positive constant $p$ satisfying $\rho(|x|)=p|x|^{-2}$ for all $(t, x) \in E$.

Proof. First, we prove (1). Substitute $r=|x|$ into the given equation and then differenciate the equation by $r$ and by $t$. We have the equations

$$
\rho^{\prime}(\nu(t) r) \nu(t)=\frac{\lambda(t)}{\nu(t)^{2}} \rho^{\prime}(r), \quad \rho^{\prime}(\nu(t) r) \nu^{\prime}(t) r=\left(\frac{\lambda^{\prime}(t)}{\nu(t)^{2}}-2 \frac{\lambda(t) \nu^{\prime}(t)}{\nu(t)^{3}}\right) \rho(r),
$$

and hence

$$
\frac{r \rho^{\prime}(r)}{\rho(r)}=\frac{\lambda^{\prime}(t) \nu(t)}{\lambda(t) \nu^{\prime}(t)}-2
$$

Therefore, both $\frac{r \rho^{\prime}(r)}{\rho(r)}$ and $\frac{\lambda^{\prime}(t) \nu(t)}{\lambda(t) \nu^{\prime}(t)}-2$ are equal to a constant $q$. Then we have

$$
\frac{\rho^{\prime}(r)}{\rho(r)}=\frac{q}{r}, \quad \frac{\lambda^{\prime}(t)}{\lambda(t)}=(q+2) \frac{\nu^{\prime}(t)}{\nu(t)} .
$$

These two equations imply

$$
\rho(r)=p r^{q}, \quad \lambda(t)=c \nu(t)^{q+2}
$$

with some positive constants $p$ and $c$. Substituting these into the given equation, we obtain $c=1$.

To prove (2), substitute $r=|x|$ into the given equation and then differentiate the equation by $r$ and by $t$, we have

$$
\rho^{\prime}\left(\frac{\nu(t)}{r}\right)=-\frac{\lambda(t) r^{5}}{\nu(t)^{3}}\left(4 \rho(r)+r \rho^{\prime}(r)\right), \quad \rho^{\prime}\left(\frac{\nu(t)}{r}\right)=\frac{\lambda(t) r^{5}}{\nu(t)^{3}}\left(\frac{\lambda^{\prime}(t) \nu(t)}{\lambda(t) \nu^{\prime}(t)}-2\right) \rho(r) .
$$

By the same argument as above, we have at first

$$
\frac{r \rho^{\prime}(r)}{\rho(r)}=-\frac{\lambda^{\prime}(t) \nu(t)}{\lambda(t) \nu^{\prime}(t)}-2=q
$$

with some constant $q$. Then

$$
\rho(r)=p r^{q}, \quad \lambda(t)=c \nu(t)^{-(q+2)}
$$

with some positive constants $p$ and $c$. Substituting these into the given equation, we obtain

$$
\rho(r)=p r^{-2}, \quad \lambda(t)=1 .
$$

The next lemma enables us to reduce the case (b) to the case (a) in the next section. 
Lemma 3. Assume that the metric $\rho$ satisfies $\rho\left(\frac{\nu}{r}\right)=\frac{\lambda r^{4}}{\nu^{2}} \rho(r)$ with some positive constants $\nu$ and $\lambda$. Then the inversion

$$
f(t, x)=\left(\lambda t, \frac{\nu x}{|x|^{2}}\right), \quad \varphi(t, x)=1
$$

is a caloric morphism from $\mathbb{R} \times\left(\mathbb{R}^{n} \backslash\{0\}\right)$ onto itself. Especially, if $\rho(r)=p r^{-2}$, then the simple inversion

$$
f(t, x)=\left(t, \frac{x}{|x|^{2}}\right), \quad \varphi(t, x)=1
$$

is a caloric morphism from $\mathbb{R} \times\left(\mathbb{R}^{n} \backslash\{0\}\right)$ onto itself.

Proof. Clearly, $(f, \varphi)$ satisfies the equations (E-1) and (E-3). To show (E-4), we have by (2) and (5),

$$
\begin{aligned}
g\left(\nabla_{g} f_{\alpha}, \nabla_{g} f_{\beta}\right) & =\frac{1}{\rho(|x|)} \frac{\nu^{2}}{|x|^{4}} \sum_{i=1}^{n}\left(\delta_{\alpha i}-2 \frac{x_{i} x_{\alpha}}{|x|^{2}}\right)\left(\delta_{\beta i}-2 \frac{x_{i} x_{\beta}}{|x|^{2}}\right) \\
& =\frac{\nu^{2}}{|x|^{4} \rho(|x|)} \delta_{\alpha \beta}, \quad \alpha, \beta=1,2, \ldots, n
\end{aligned}
$$

By the assumption on $\rho$, we have

$$
\begin{aligned}
\frac{\nu^{2}}{|x|^{4} \rho(|x|)} \delta_{\alpha \beta} & =\lambda \delta_{\alpha \beta} \frac{1}{\rho(\nu /|x|)} \\
& =f_{0}^{\prime} \cdot g^{\alpha \beta} \circ f, \quad \alpha, \beta=1,2, \ldots, n .
\end{aligned}
$$

Finally, we shall show (E-2). By (1) and (3), we have for $\alpha=1,2, \ldots, n$,

$$
\begin{aligned}
\Delta_{g} f_{\alpha}+2 g\left(\nabla_{g} \log \varphi, \nabla_{g} f_{\alpha}\right)-\frac{d f_{0}}{d t}\left[\left(\Delta_{g} x_{\alpha}\right) \circ f\right] \\
=\frac{1}{\rho(r)} 2(2-n) \frac{\nu x_{\alpha}}{r^{4}}+\frac{n-2}{2} \frac{\rho^{\prime}(r)}{\rho(r)^{2}} \sum_{i=1}^{n} \frac{x_{i}}{r}\left(\frac{\nu \delta_{i \alpha}}{r^{2}}-2 \frac{\nu x_{i} x_{\alpha}}{r^{4}}\right) \\
\quad+\lambda \frac{2-n}{2} \frac{\rho^{\prime}(\nu / r)}{\rho(\nu / r)^{2}} \frac{\nu x_{\alpha} / r^{2}}{\nu / r} \\
=\frac{2-n}{2}\left(\frac{4}{r^{4} \rho(r)}+\frac{\rho^{\prime}(r)}{r^{3} \rho(r)^{2}}\right) \nu x_{\alpha}+\lambda \frac{2-n}{2} \frac{\rho^{\prime}(\nu / r)}{\rho(\nu / r)^{2} r} x_{\alpha}
\end{aligned}
$$

where we put $r=|x|$ for simplicity. On the other hand, the assumption on $\rho$ implies

$$
\frac{\rho^{\prime}(\nu / r)}{\rho(\nu / r)^{2} r}=\frac{\nu r}{\lambda}\left(\frac{1}{r^{4}} \frac{1}{\rho(r)}\right)^{\prime}=-\frac{\nu}{\lambda}\left(\frac{4}{r^{4} \rho(r)}+\frac{\rho^{\prime}(r)}{r^{3} \rho(r)^{2}}\right)
$$

because $\frac{\rho^{\prime}(\nu / r)}{\rho(\nu / r)^{2} r}=\frac{r}{\nu}\left(\frac{1}{\rho(\nu / r)}\right)^{\prime}$. Thus we have

$$
\Delta_{g} f_{\alpha}+2 g\left(\nabla_{g} \log \varphi, \nabla_{g} f_{\alpha}\right)-\frac{d f_{0}}{d t}\left[\left(\Delta_{g} x_{\alpha}\right) \circ f\right]=0=\frac{\partial f_{\alpha}}{\partial t} .
$$

Therefore $(f, \varphi)$ is a caloric morphism.

We close this section with the following lemma concerning the equation for the function $\log \varphi$. 
Lemma 4. Let $(f, \varphi)$ be a caloric morphism such that $f$ is of form (a).

(1) $\varphi$ satisties the following equation:

$$
\frac{\partial \log \varphi}{\partial x_{j}}=\frac{\rho(|x|)}{2}\left(\frac{\nu^{\prime}(t)}{\nu(t)} x_{j}+\sum_{k=1}^{n} s_{j k}(t) x_{k}\right), \quad j=1,2, \ldots, n,
$$

where $s_{j k}(t)(j, k=1, \ldots, n)$ is the $(j, k)$-element of the skew symmetric matrix

$$
S(t)=R(t)^{-1} R^{\prime}(t)
$$

(2) Unless both the coditions $n=2$ and $\rho(r)=p r^{-2}$ hold, $R^{\prime}(t)=O$ holds for all $t$. In this case, the equation (8) becomes

$$
\frac{\partial \log \varphi}{\partial x_{j}}=\frac{\rho(|x|)}{2} \frac{\nu^{\prime}(t)}{\nu(t)} x_{j}, \quad j=1,2, \ldots, n .
$$

Proof. Since $f(t, x)=\left(f_{0}(t), \nu(t) R(t) x\right)$, where $\nu(t)>0$ and $R(t)$ is an orthogonal matrix, we have

$$
\begin{aligned}
\Delta_{g} f_{\alpha} & =\frac{1}{\rho(|x|)} \Delta_{x} f_{\alpha}+\frac{n-2}{2} \frac{\rho^{\prime}(|x|)}{\rho(|x|)^{2}} \sum_{i=1}^{n} \frac{x_{i}}{|x|} \frac{\partial f_{\alpha}}{\partial x_{i}} \\
& =\frac{n-2}{2} \frac{\rho^{\prime}(|x|)}{|x| \rho(|x|)^{2}} \nu(t) \sum_{i=1}^{n} r_{\alpha i}(t) x_{i}
\end{aligned}
$$

and

$$
\begin{aligned}
2 g\left(\nabla_{g} \log \varphi, \nabla_{g} f_{\alpha}\right) & =\frac{2}{\rho(|x|)} \sum_{i=1}^{n} \frac{\partial \log \varphi}{\partial x_{i}} \frac{\partial f_{\alpha}}{\partial x_{i}} \\
& =\frac{2}{\rho(|x|)} \sum_{i=1}^{n} \frac{\partial \log \varphi}{\partial x_{i}} \nu(t) r_{\alpha i}(t)
\end{aligned}
$$

for $\alpha=1,2, \ldots, n$ by (1), (2) and (4). The formula (3) implies

$$
\begin{aligned}
f_{0}^{\prime}\left[\left(\Delta_{g} x_{\alpha}\right) \circ f\right] & =f_{0}^{\prime}(t) \frac{n-2}{2} \frac{\rho^{\prime}(|f|)}{\rho(|f|)^{2}} \frac{f_{\alpha}}{|f|} \\
& =f_{0}^{\prime}(t) \frac{n-2}{2} \frac{\rho^{\prime}(|f|)}{\rho(|f|)^{2}} \frac{\sum_{i=1}^{n} \nu(t) r_{\alpha i}(t) x_{i}}{\nu(t)|x|} \\
& =f_{0}^{\prime}(t) \frac{n-2}{2} \frac{\rho^{\prime}(\nu(t)|x|)}{|x| \rho(\nu(t)|x|)^{2}} \sum_{i=1}^{n} r_{\alpha i}(t) x_{i}
\end{aligned}
$$

for $\alpha=1,2, \ldots, n$. On the other hand, differentiating (6) by $|x|$, we have

$$
\frac{f_{0}^{\prime}(t) \rho^{\prime}(\nu(t)|x|)}{\rho(\nu(t)|x|)^{2}}=\frac{\nu(t) \rho^{\prime}(|x|)}{\rho(|x|)^{2}} .
$$


Substituting (4), (10), (11), (12) and (13) into (E-2'):

$$
\frac{\partial f_{\alpha}}{\partial t}=\Delta_{g} f_{\alpha}+2 g\left(\nabla_{g} \log \varphi, \nabla_{g} f_{\alpha}\right)-f_{0}^{\prime}\left[\left(\Delta_{g} x_{\alpha}\right) \circ f\right],
$$

we have

$$
\sum_{k=1}^{n}\left(\nu^{\prime}(t) r_{\alpha k}(t)+\nu(t) r_{\alpha k}^{\prime}(t)\right) x_{k}=\frac{2}{\rho(|x|)} \sum_{i=1}^{n} \frac{\partial \log \varphi}{\partial x_{i}} \nu(t) r_{\alpha i}(t) .
$$

Therefore, the equation

$$
\frac{\partial \log \varphi}{\partial x_{j}}=\frac{\rho(|x|)}{2}\left(\frac{\nu^{\prime}(t)}{\nu(t)} x_{j}+\sum_{k=1}^{n} s_{j k}(t) x_{k}\right), \quad j=1,2, \ldots, n
$$

holds, where $s_{j k}(t)$ is the $(j, k)$ element of the matrix $S(t)=R(t)^{-1} R^{\prime}(t)$.

Next we proceed to prove the statement (2). Differentiating the equation (14) by $x_{i}(i \neq j)$, we have

$$
\frac{\partial}{\partial x_{i}} \frac{\partial \log \varphi}{\partial x_{j}}=\frac{\rho^{\prime}(|x|)}{2|x|}\left(\frac{\nu^{\prime}(t)}{\nu(t)} x_{i} x_{j}+\sum_{l=1}^{n} s_{j l}(t) x_{i} x_{l}\right)+\frac{\rho(|x|)}{2} s_{j i}(t) .
$$

Also

$$
\frac{\partial}{\partial x_{j}} \frac{\partial \log \varphi}{\partial x_{i}}=\frac{\rho^{\prime}(|x|)}{2|x|}\left(\frac{\nu^{\prime}(t)}{\nu(t)} x_{j} x_{i}+\sum_{l=1}^{n} s_{i l}(t) x_{j} x_{l}\right)+\frac{\rho(|x|)}{2} s_{i j}(t)
$$

holds. Since $\frac{\partial}{\partial x_{i}} \frac{\partial \log \varphi}{\partial x_{j}}=\frac{\partial}{\partial x_{j}} \frac{\partial \log \varphi}{\partial x_{i}}$

$$
\frac{\rho^{\prime}(|x|)}{|x|} \sum_{l=1}^{n} s_{j l}(t) x_{i} x_{l}+\rho(|x|) s_{j i}(t)=\frac{\rho^{\prime}(|x|)}{|x|} \sum_{l=1}^{n} s_{i l}(t) x_{j} x_{l}+\rho(|x|) s_{i j}(t),
$$

holds for all $i, j=1,2, \ldots, n$ with $i \neq j$. The matrix $S(t)$ is skew symmetric because $R(t)$ is orthogonal. Then we have

$$
2 s_{i j}(t)=\frac{\rho^{\prime}(|x|)}{2|x| \rho(|x|)}\left(x_{i} \sum_{l=1}^{n} s_{j l}(t) x_{l}-x_{j} \sum_{l=1}^{n} s_{i l}(t) x_{l}\right)
$$

for all $i, j=1,2, \ldots, n$ with $i \neq j$, and hence

$$
s_{i j}(t)=\frac{\rho^{\prime}(|x|)}{2|x| \rho(|x|)}\left(x_{i} y_{j}-y_{i} x_{j}\right)
$$

where we put $y=S x$. 
First we consider the case that $n \geqq 3$. Then for each fixed $t$ and each triple indices $i, j, k$ with $1 \leqq i<j<l \leqq n$, the equation (15) implies

$$
s_{i j}(t) x_{l}+s_{j l}(t) x_{i}+s_{l i}(t) x_{j}=0
$$

for all $\left(x_{i}, x_{j}, x_{l}\right)$ in a open subset of $\mathbb{R}^{3}$. That implies $\left(s_{i j}(t), s_{j l}(t), s_{l i}(t)\right)=0$ for each $1 \leqq i<j<l \leqq n$. Therefore $S(t)=O$ and hence $R^{\prime}(t)=O$ for all $t$, if $n \geqq 3$.

Next, in the case that $n=2$, the equation (16) implies

$$
s_{12}(t)=-\frac{r \rho^{\prime}(r)}{2 \rho(r)} s_{12}(t)
$$

Therefore $s_{12}=0$ unless $\rho(r)=p r^{-2}$. Thus we have shown that $R^{\prime}(t)=O$ for all $t$ unless $n=2$ and $\rho(r)=p r^{-2}$. Consequently, the equation (9) immediately follows from (8) and $S(t)=O$.

\section{Some special cases.}

Before the proof of Theorem 1, we deal with the case that $\rho$ has the form $\rho(r)=p r^{q}$ in this section. The following theorem corresponds to the cases 1-a and $1-\mathrm{b}$ of Theorem 1 .

Theorem 2. Let $n=2$ and $\rho(r)=\frac{p}{r^{2}}(p>0)$.

(1) Let $(f, \varphi)$ be a caloric morphism such that $f$ is of form (a). Then

$$
\begin{aligned}
f(t, x) & =\left(t+d, c e^{a t} R_{0}\left(\begin{array}{cc}
\cos b t & -\sin b t \\
\sin b t & \cos b t
\end{array}\right) x\right), \\
\varphi(t, r, \theta) & =C r^{\frac{1}{2} a p} \exp \left(\frac{p}{2} b \theta+\frac{p}{4}\left(a^{2}+b^{2}\right) t\right) .
\end{aligned}
$$

(2) Let $(f, \varphi)$ be a caloric morphism such that $f$ is of form (b). Then

$$
\begin{aligned}
f(t, x) & =\left(t+d, \frac{c e^{a t}}{|x|^{2}} R_{0}\left(\begin{array}{cc}
\cos b t & -\sin b t \\
\sin b t & \cos b t
\end{array}\right) x\right), \\
\varphi(t, r, \theta) & =C r^{-\frac{1}{2} a p} \exp \left(\frac{p}{2} b \theta+\frac{p}{4}\left(a^{2}+b^{2}\right) t\right) .
\end{aligned}
$$

In both cases, $a, b, c, d, C \in \mathbb{R}(c, C>0), R_{0}$ is an orthogonal matrix and $(r, \theta)$ is. the polar coordinate of $\mathbb{R}^{2}$. $a=0$ if and only if $\nu^{\prime}(t)=0$ for some $t$, where $\nu$ is the function appeared in the forms (a) and (b).

Proof. First of all, $f_{0}^{\prime}(t)=1$ follows from the equality (6) in Lemma 1 . Therefore $f_{0}(t)=t+d$ with $d \in \mathbb{R}$.

Next, by Lemma $4(1), \log \varphi$ satisfies the equation

$$
\frac{\partial \log \varphi}{\partial x_{j}}=\frac{p|x|^{-2}}{2}\left(\frac{\nu^{\prime}(t)}{\nu(t)} x_{j}+\sum_{k=1}^{2} s_{j k}(t) x_{k}\right)
$$


for each $j=1,2$. Since $S(t)$ is skew symmetric and $n=2, S(t)=\left(\begin{array}{cc}0 & -s(t) \\ s(t) & 0\end{array}\right)$ where we put $s(t)=s_{21}(t)$ for simplicity. Then (16) becomes

$$
\begin{aligned}
& \frac{\partial \log \varphi}{\partial x_{1}}=\frac{p}{2|x|^{2}}\left(\frac{\nu^{\prime}(t)}{\nu(t)} x_{1}-s(t) x_{2}\right) \\
& \frac{\partial \log \varphi}{\partial x_{2}}=\frac{p}{2|x|^{2}}\left(\frac{\nu^{\prime}(t)}{\nu(t)} x_{2}+s(t) x_{1}\right) .
\end{aligned}
$$

In the polar coordinate $x_{1}=r \cos \theta, x_{2}=r \sin \theta,(17)$ becomes

$$
\begin{aligned}
& \frac{\partial \log \varphi}{\partial r}=\frac{x_{1}}{r} \frac{\partial \log \varphi}{\partial x_{1}}+\frac{x_{2}}{r} \frac{\partial \log \varphi}{\partial x_{2}}=\frac{p}{2} \frac{\nu^{\prime}(t)}{\nu(t)} \frac{1}{r} \\
& \frac{\partial \log \varphi}{\partial \theta}=-x_{2} \frac{\partial \log \varphi}{\partial x_{1}}+x_{1} \frac{\partial \log \varphi}{\partial x_{2}}=\frac{p}{2} s(t) .
\end{aligned}
$$

Integrating these equations, we have

$$
\log \varphi(t, r, \theta)=\frac{p}{2} \frac{\nu^{\prime}(t)}{\nu(t)} \log r+\frac{p}{2} s(t) \theta+\psi(t) .
$$

On the other hand, $\varphi$ satisfies the equation (E-1): $H_{g} \varphi=0$. We put $w=\log \varphi$ for simplicity. Since

$$
\frac{\partial \varphi}{\partial t}-\Delta_{g} \varphi=\varphi\left\{\frac{\partial w}{\partial t}-\frac{1}{\rho}\left[\Delta_{x} w+\left|\nabla_{x} w\right|^{2}+\frac{n-2}{2} \frac{\rho^{\prime}}{\rho} \frac{\partial w}{\partial r}\right]\right\}
$$

holds, $w$ satisfies the equation

$$
\frac{\partial w}{\partial t}-\frac{1}{\rho}\left[\frac{\partial^{2} w}{\partial r^{2}}+\left(\frac{\partial w}{\partial r}\right)^{2}+\left(\frac{1}{r} \frac{\partial w}{\partial \theta}\right)^{2}+\frac{1}{r} \frac{\partial w}{\partial r}+\frac{1}{r^{2}} \frac{\partial^{2} w}{\partial \theta^{2}}\right]=0 .
$$

Differentiating (18), we have

$$
\begin{gathered}
\frac{\partial w}{\partial t}=\frac{p}{2}\left(\frac{\nu^{\prime}}{\nu}\right)^{\prime} \log r+\frac{p}{2} s^{\prime} \theta+\psi^{\prime} \\
\frac{\partial w}{\partial r}=\frac{p}{2 r} \frac{\nu^{\prime}}{\nu}, \quad\left(\frac{\partial w}{\partial r}\right)^{2}=\frac{p^{2}}{4 r^{2}}\left(\frac{\nu^{\prime}}{\nu}\right)^{2}, \quad \frac{\partial^{2} w}{\partial r^{2}}=-\frac{p}{2 r^{2}} \frac{\nu^{\prime}}{\nu} \\
\left(\frac{1}{r} \frac{\partial w}{\partial \theta}\right)^{2}=\frac{p^{2}}{4 r^{2}} s^{2} \quad \text { and } \quad \frac{\partial^{2} w}{\partial \theta^{2}}=0
\end{gathered}
$$

Substitute these calculations into (20). Then we have the equation

$$
\frac{p}{2}\left(\frac{\nu^{\prime}}{\nu}\right)^{\prime} \log r+\frac{p}{2} s^{\prime} \theta+\psi^{\prime}-\frac{p}{4}\left[\left(\frac{\nu^{\prime}}{\nu}\right)^{2}+s^{2}\right]=0
$$


Therefore we obtain a system of differential equations

$$
\left\{\begin{array}{l}
\left(\frac{\nu^{\prime}}{\nu}\right)^{\prime}=0 \\
s^{\prime}=0 \\
\psi^{\prime}=\frac{p}{4}\left[\left(\frac{\nu^{\prime}}{\nu}\right)^{2}+s^{2}\right]
\end{array}\right.
$$

by (21). The solution of this system is

$$
\left\{\begin{array}{l}
\nu(t)=c e^{a t} \\
s(t)=b \\
\psi(t)=\frac{p}{4}\left(a^{2}+b^{2}\right) t+C_{0}
\end{array}\right.
$$

where $a, b, c, C_{0} \in \mathbb{R}, a \neq 0$ and $c>0$. Clearly, $a=0$ if and only if $\nu^{\prime}(t)=0$ for all $t$. Substituting (22) into (18), we have

$$
\log \varphi(t, r, \theta)=\frac{a p}{2} \log r+\frac{p}{2} b \theta+\frac{p}{4}\left(a^{2}+b^{2}\right) t+C
$$

and

$$
S(t)=\left(\begin{array}{cc}
0 & -b \\
b & 0
\end{array}\right)
$$

Therefore

$$
\varphi(t, r, \theta)=C r^{\frac{1}{2} a p} \exp \left(\frac{p}{2} b \theta+\frac{p}{4}\left(a^{2}+b^{2}\right) t\right)
$$

Now choose a number $t_{0} \in \mathbb{R}$ so that $\left\{t=t_{0}\right\} \cap D \neq \emptyset$, and put $R_{0}=R\left(t_{0}\right)$. Since $R(t)$ is continuous, we can put

$$
R(t)=R_{0}\left(\begin{array}{cc}
\cos v(t) & -\sin v(t) \\
\sin v(t) & \cos v(t)
\end{array}\right)
$$

where $v(t)$ is a $C^{\infty}$-function such that $v\left(t_{0}\right)=0$. Substituting this into the definition of $S(t)$, we have

$$
\begin{aligned}
S(t) & =\left(\begin{array}{cc}
\cos v(t) & \sin v(t) \\
-\sin v(t) & \cos v(t)
\end{array}\right){ }^{t} R_{0} R_{0}\left(\begin{array}{cc}
-\sin v(t) & -\cos v(t) \\
\cos v(t) & -\sin v(t)
\end{array}\right) v^{\prime}(t) \\
& =\left(\begin{array}{cc}
0 & -1 \\
1 & 0
\end{array}\right) v^{\prime}(t) .
\end{aligned}
$$

Then by (23), we have

$$
\left(\begin{array}{cc}
0 & -b \\
b & 0
\end{array}\right)=\left(\begin{array}{cc}
0 & -1 \\
1 & 0
\end{array}\right) v^{\prime}(t)
$$


and hence $v(t)=b t$. Thus

$$
\begin{aligned}
f(t, x) & =\left(t+d, c e^{a t} R_{0}\left(\begin{array}{cc}
\cos b t & -\sin b t \\
\sin b t & \cos b t
\end{array}\right) x\right) \\
\varphi(t, r, \theta) & =C r^{\frac{1}{2} a p} \exp \left(\frac{p}{2} b \theta+\frac{p}{4}\left(a^{2}+b^{2}\right) t\right)
\end{aligned}
$$

for all $(t, x) \in D$. We have shown the first statement (1). The proof of the second statement is reduced to the first statement by the composition with an inversion. In fact, $\rho(r)=p r^{-2}$ satisfies $\rho\left(\frac{1}{r}\right)=r^{4} \rho(r)$. Then the inversion $(j, 1)$, where

$$
j(t, x)=\left(t, \frac{x}{|x|^{2}}\right)
$$

is a caloric morphism by Lemma 3. The composition $(f \circ j, \varphi \circ j)$ of $(f, \varphi)$ and $(j, 1)$ is

$$
\left(f_{0}(t), \nu(t) R(t) x\right), \quad \varphi\left(t, \frac{x}{|x|^{2}}\right)
$$

which is reduced to the case that $f \circ j$ is of form (a). Thus we have shown Theorem 2.

The next theorem corresponds to the cases 2-a and 2-b of Theorem 1.

Theorem 3. Let $n \geqq 3$ and $\rho$ is of form $\rho(r)=\frac{p}{r^{2}}(p>0)$.

(1) Let $(f, \varphi)$ be a caloric morphism such that $f$ is of form (a). Then

$$
f(t, x)=\left(t+d, c e^{a t} R_{0} x\right), \quad \varphi(t, x)=C|x|^{\frac{1}{2} a p} \exp \left(\frac{1}{4} p a^{2} t\right) .
$$

(2) Let $(f, \varphi)$ be a caloric morphism such that $f$ is of form (b). Then

$$
f(t, x)=\left(t+d, \frac{c e^{a t}}{|x|^{2}} R_{0} x\right), \quad \varphi(t, x)=C|x|^{-\frac{1}{2} a p} \exp \left(\frac{1}{4} p a^{2} t\right) .
$$

In both cases, $a, c, d, C \in \mathbb{R}(c, C>0), R_{0}$ is an orthogonal matrix. $a=0$ if and only if $\nu^{\prime}(t)=0$ for some $t$, where $\nu$ is the function appeared in the forms (a) and (b).

Proof. First of all, $f_{0}^{\prime}(t)=1$ follows from the equality (6) in Lemma 1. Therefore $f_{0}(t)=t+d$ with $d \in \mathbb{R}$.

By Lemma 4 (2), $R$ is constant and $\log \varphi$ satisfies the equation

$$
\frac{\partial \log \varphi}{\partial x_{j}}=\frac{p}{2|x|^{2}} \frac{\nu^{\prime}(t)}{\nu(t)} x_{j}, \quad j=1, \ldots, n,
$$

because $n>2$. It follows that $\varphi$ is a radial function of $x$, i.e.

$$
\varphi(t, x)=\varphi(t,|x|)
$$


and that

$$
\frac{\partial \log \varphi}{\partial r}=\frac{p \nu^{\prime}(t)}{2 \nu(t)} \frac{1}{r}
$$

and hence

$$
\log \varphi(t, r)=\frac{p \nu^{\prime}(t)}{2 \nu(t)} \log r+\psi(t) .
$$

By (E-1): $H_{g} \varphi=0$ and (19), $w=\log \varphi$ satisfies the equation

$$
\frac{\partial w}{\partial t}-\frac{1}{\rho}\left[\frac{\partial^{2} w}{\partial r^{2}}+\left(\frac{\partial w}{\partial r}\right)^{2}+\frac{1}{r} \frac{\partial w}{\partial r}\right]=0 .
$$

Substituting the following

$$
\begin{gathered}
\frac{\partial w}{\partial t}=\frac{p}{2}\left(\frac{\nu^{\prime}}{\nu}\right)^{\prime} \log r+\psi^{\prime} \\
\frac{\partial w}{\partial r}=\frac{p}{2 r} \frac{\nu^{\prime}}{\nu}, \quad\left(\frac{\partial w}{\partial r}\right)^{2}=\frac{p^{2}}{4 r^{2}}\left(\frac{\nu^{\prime}}{\nu}\right)^{2}, \quad \frac{\partial^{2} w}{\partial r^{2}}=-\frac{p}{2 r^{2}} \frac{\nu^{\prime}}{\nu}
\end{gathered}
$$

into (25), we have the equation

$$
\frac{p}{2}\left(\frac{\nu^{\prime}}{\nu}\right)^{\prime} \log r+\psi^{\prime}-\frac{p}{4}\left(\frac{\nu^{\prime}}{\nu}\right)^{2}=0 .
$$

Therefore we obtain a system of differential equations

$$
\left\{\begin{array}{l}
\left(\frac{\nu^{\prime}}{\nu}\right)^{\prime}=0 \\
\psi^{\prime}=\frac{p}{4}\left(\frac{\nu^{\prime}}{\nu}\right)^{2}
\end{array}\right.
$$

The solution is

$$
\left\{\begin{array}{l}
\nu(t)=c e^{a t} \\
\psi(t)=\frac{p a^{2}}{4} t+C_{0}
\end{array}\right.
$$

where $a, c, C_{0} \in \mathbb{R}, a \neq 0$ and $c>0$. Clearly, $a=0$ if and only if $\nu^{\prime}(t)=0$ for some $t$. Substituting (26) into (24), we have

$$
\log \varphi(t, r)=\frac{a p}{2} \log r+\frac{p a^{2}}{4} t+C_{0}
$$

Thus we have,

$$
f(t, x)=\left(t+d, c e^{a t} R x\right), \quad \varphi(t, x)=C|x|^{\frac{1}{2} a p} \exp \left(\frac{1}{4} p a^{2} t\right)
$$

for all $(t ; x) \in D$. We have shown the first statement (1). As in Theorem 2 , the proof of the second statement is reduced to the first statement by composing the inversion $(j, 1)$.

The next theorem corresponds to the case 3 of Theorem 1 . We note that the case of $d / a--1, b=c=0, p=1$ and $q=0$ is the Appell transformation. 
Theorem 4. Let $\rho$ be of form $\rho(r)=p r^{q}(p>0, q \in \mathbb{R}, q \neq-2)$ and let $(f, \varphi)$ be a caloric morphism such that $f$ is of form (a) or (b). Then

$$
\begin{aligned}
& f(t, x)=\left(\frac{c t+d}{a t+b}, \frac{R_{0} x}{|a t+b|^{2 /(q+2)}}\right), \\
& \varphi(t, x)=\frac{C}{|a t+b|^{n / 2}} \exp \left[-\frac{p a|x|^{q+2}}{(q+2)^{2}(a t+b)}\right],
\end{aligned}
$$

where $a, b, c, d, C \in \mathbb{R}(b c-a d=1, C>0)$ and $R_{0}$ is an orthogonal matrix. $a=0$ if and only if $\nu^{\prime}(t)=0$ for some $t$, where $\nu$ is the function appeared in the forms (a) and (b).

Proof. Let $q \neq-2$. Then by Lemma 4 (2), $R$ is constant and

$$
\frac{\partial \log \varphi}{\partial x_{j}}=\frac{p|x|^{q}}{2} \frac{\nu^{\prime}(t)}{\nu(t)} x_{j}, \quad j=1, \ldots, n
$$

holds. As in the proof of Theorem $4, \varphi$ is a radial function of $x, \varphi(t, x)=\varphi(t,|x|)$ and hence

$$
\log \varphi(t, r)=\frac{p r^{q+2}}{2(q+2)} \frac{\nu^{\prime}(t)}{\nu(t)}+\psi(t), \quad r=|x|
$$

By (E-1) and (19), $w=\log \varphi$ satisfies the equation

$$
\frac{\partial w}{\partial t}-\frac{1}{\rho}\left[\frac{\partial^{2} w}{\partial r^{2}}+\left(\frac{\partial w}{\partial r}\right)^{2}+\frac{n q+2 n-2 q-2}{2 r} \frac{\partial w}{\partial r}\right]=0 .
$$

Differentiating (27), we have

$$
\begin{gathered}
\frac{\partial w}{\partial t}=\frac{p r^{q+2}}{2(q+2)}\left(\frac{\nu^{\prime}}{\nu}\right)^{\prime}+\psi^{\prime}, \quad \frac{\partial w}{\partial r}=\frac{p r^{q+1}}{2} \frac{\nu^{\prime}}{\nu} \\
\left(\frac{\partial w}{\partial r}\right)^{2}=\frac{p^{2} r^{2 q+2}}{4}\left(\frac{\nu^{\prime}}{\nu}\right)^{2}, \quad \frac{\partial^{2} w}{\partial r^{2}}=\frac{(q+1) p r^{q}}{2} \frac{\nu^{\prime}}{\nu}
\end{gathered}
$$

Substitute these into (28). Then we have

$$
\frac{p r^{q+2}}{2(q+2)}\left(\frac{\nu^{\prime}}{\nu}\right)^{\prime}+\psi^{\prime}-\left[\frac{p r^{q+2}}{4}\left(\frac{\nu^{\prime}}{\nu}\right)^{2}+\frac{n q+2 n}{4} \frac{\nu^{\prime}}{\nu}\right]=0
$$

and hence

$$
\frac{p r^{q+2}}{2(q+2)}\left[\left(\frac{\nu^{\prime}}{\nu}\right)^{\prime}-\frac{q+2}{2}\left(\frac{\nu^{\prime}}{\nu}\right)^{2}\right]+\psi^{\prime}-\frac{n(q+2)}{4}(\log \nu)^{\prime}=0 .
$$

Therefore we obtain a system of differential equations,

$$
\left\{\begin{array}{l}
\left(\frac{\nu^{\prime}}{\nu}\right)^{\prime}-\frac{q+2}{2}\left(\frac{\nu^{\prime}}{\nu}\right)^{2}=0 \\
\psi^{\prime}-\frac{n(q+2)}{4}(\log \nu)^{\prime}=0
\end{array}\right.
$$


The solution is

$$
\left\{\begin{array}{l}
\nu(t)=\frac{1}{|a t+b|^{2 /(q+2)}}, \\
\psi(t)=\log C \nu^{n(q+2) / 4}=\log C|a t+b|^{-n / 2}
\end{array}\right.
$$

where $a, b \in \mathbb{R}$ and $C>0$. Clearly, $a=0$ if and only if $\nu^{\prime}(t)=0$ for some $t$. Substituting (29) into (27), we have

$$
\log \varphi(t, r)=-\frac{p a r^{q+2}}{(q+2)^{2}(a t+b)}+\log C|a t+b|^{-n / 2}
$$

On the other hand, (6) in Lemma 1 implies

$$
f_{0}^{\prime}(t)=\nu^{q+2}(t)=\frac{1}{|a t+b|^{2}}
$$

Therefore $f_{0}(t)=\frac{c t+d}{a t+b}$ where $a, b, c, d \in \mathbb{R}$ and $b c-a d=1$. Consequently,

$$
\begin{aligned}
f(t, x) & =\left(\frac{c t+d}{a t+b}, \frac{R_{0} x}{|a t+b|^{2 /(q+2)}}\right) \\
\varphi(t, x) & =\frac{C}{|a t+b|^{n / 2}} \exp \left[-\frac{p a|x|^{q+2}}{(q+2)^{2}(a t+b)}\right]
\end{aligned}
$$

for all $(t, x) \in D$. Thus we have shown Theorem 4 .

\section{Proof of Theorem 1.}

Now we can prove our main theorem.

Proof of Theorem 1. First we deal with the case that the function $\nu(t)$ is not constant. Let $I_{0}$ be the open interval consisting of all $t$ such that $(t, x) \in D$ for some $x \in \mathbb{R}^{n}$. Let $I$ be a connected component of the open set $\left\{t \in I_{0} ; \nu^{\prime}(t) \neq 0\right\}$ and let $J$ be the open interval consisting of all $|x|$ such that $(t, x) \in D$ for some $t \in I$. Then $\rho(r)=p r^{q}$ on $J$ by Lemma 2 , and all the cases have already been treated in the theorems, Theorem 2, 3 and 4. Since we assume that $\nu$ is not constant, if $q=-2$,

$$
\nu^{\prime}(t)=c a e^{a t}, \quad(a \neq 0)
$$

on $I$ from Theorem 2 and Theorem 3 , and if $q \neq-2$,

$$
\nu^{\prime}(t)=\frac{-2 a}{(q+2)|a t+b|^{2 /(q+2)}}, \quad(a \neq 0)
$$

on $I$ from Theorem 4 . In both cases, $\nu^{\prime}(t) \neq 0$ on $I$, the closure of $I$ in $I_{0}$. Hence, $I=I_{0}$ because $I_{0}$ is connected. Therefore $D \subset I \times J$ and $\rho(r)$ has the form $p r^{q}$. Thus we have shown that $(f, \varphi)$ and $\rho$ are completely determined as the cases 1-a, 1-b, 2-a, 2-b, 3 in Theorem 1, if the function $\nu(t)$ is not constant. 
Next we deal with the case that $\nu$ is constant. Because of the preceding argument, we may exclude the case that $\rho(r)$ has the form $\rho(r)=p r^{q}$. We first consider the case (a) as before. By Lemma $4, R^{\prime}(t)=O$ and

$$
\frac{\partial \log \varphi}{\partial x_{j}}=0
$$

because $\nu^{\prime}(t)=0$. Therefore $R$ is a constant matrix $R_{0}$ and $\varphi$ depends only on $t$. Since $\varphi$ satisfies (E-1): $H_{g} \varphi=\frac{\partial \varphi}{\partial t}=0, \varphi$ is a positive constant $C$. On the other hand, (6) in Lemma 1 implies $\rho(\nu r)=f_{0}^{\prime}(t) \rho(r)$. Therefore $f_{0}^{\prime}(t)=\rho(\nu r) / \rho(r)$ and hence $f_{0}^{\prime}=\lambda$ with some positive constant $\lambda$. Thus we have

$$
f(t, x)=\left(\lambda t+d, \nu R_{0} x\right), \quad \varphi(t, x)=C
$$

This is Case 4 .

Finally, we consider the case (b). Since $\nu$ is constant, $f_{0}^{\prime}$ is equal to a constant $\lambda$ such that

$$
\rho\left(\frac{\nu}{r}\right)=\frac{\lambda r^{4}}{\nu^{2}} \rho(r)
$$

by (7) in Lemma 1. Then, the inversion $(h, 1)$, where

$$
h(t, x)=\left(\lambda t, \frac{\nu x}{|x|^{2}}\right)
$$

is a caloric morphism by Lemma 3 . The composition of $(h, 1)$ and $(f, \varphi)$ is

$$
\left(\lambda f_{0}(t), R x\right), \quad \varphi(t, x)
$$

which is reduced to the case that $f \circ h$ is of form (a). Thus we have completed the proof of Theorem 1 .

\section{REFERENCES}

[1] B. Fuglede, Harmonic Morphisms Between Riemannian Manifolds, Ann. Inst. Fourier, Grenoble 28 (1978), 107-144.

[2] T. Ishihara, A mapping of Riemannian manifolds which preserves harmonic functions, $\mathrm{J}$. Math. Kyoto Univ. 19 (1979), 215-229.

[3] H. Leutwiler, On the Appell Transformation, Potential Theory (J.Král, J.Lukeš, I.Netuka, J,Veselý, eds.), Plenum, New York, 1988, pp. 215-222.

[4] M. Nishio and K. Shimomura, Caloric morphisms on semi-euclidean space, Rev. Roumaine. Math. Pures Appl. 47 (2002), 727-736.

[5] M. Nishio and K. Shimomura, A charactorization of caloric morphisms between manifolds, Ann. Acad. Sci. Fenn. Math. 28 (2003), 111-122.

[6] K. Shimomura, The determination of caloric morphisms on Euclidean domains, Nagoya Math. J. 158 (2000), 133-166. 